\title{
Verschiedene Befragungsmodi, unterschiedliche Ergebnisse? Moduseffekte und soziale Erwünschtheit am Beispiel Wahl der FPÖ
}

\author{
Julian Aichholzer ${ }^{1, *}$ \\ 1 Institut für Staatswissenschaft der Universität Wien, Austria \\ * julian.aichholzer@univie.ac.at
}

\section{Zusammenfassung}

Der zunehmende Einsatz von Online-Befragungen (CAWI) ist begleitet von der Frage nach "Moduseffekten“ bzw. Unterschieden in substanziellen Ergebnissen. Diese Studie fokussiert auf die mögliche Erklärung von Moduseffekten in der Wahlforschung am Beispiel der berichteten Wahlentscheidung für die FPÖ. Ziel der Analyse mit Daten der AUTNES 2013 und einer Gegenüberstellung von CAWI mit CAPI (face-to-face) ist die Zerlegung indirekter Effekte des Erhebungsmodus auf die Parteiwahl. Die Ergebnisse legen nahe, dass Unterschiede in der Wahrscheinlichkeit der Wahl für die FPÖ zwischen CAWI und CAPI kaum auf soziodemografische Heterogenität zurückführen sind, sondern deuten auf die stärkere Inklusion politisch Entfremdeter und geringere Effekte sozialer Erwünschtheit in der generellen Äußerung ideologisch rechter Einstellungen im CAWI-Modus hin.

\section{Schlüsselwörter}

Umfragen, Erhebungsmodus, Internet-Panels, Wahlforschung FPÖ

\section{Different survey modes, different results? Mode effects and social desirability in the vote for the FPÖ}

\begin{abstract}
The increasing use of online surveys (CAWI) raises the question about "mode effects", i.e., substantive differences in results. This study looks at causes of mode effects in electoral research, taking reported vote choice for the FPÖ as an example. For this purpose, data from AUTNES 2013 surveys are used to decompose indirect effects of the survey mode on vote choice, comparing CAWI with CAPI (face-to-face). The results suggest that differences in the likelihood of voting for the FPÖ in CAWI and CAPI are hardly related to socio-demographic heterogeneity, but rather result from including more politically alienated people and generally from less social desirability when expressing right-wing ideological attitudes in the CAWI mode.
\end{abstract}

\section{Keywords}

surveys, survey mode, internet panels, electoral research, FPÖ

The author has declared that no competing interests exist. 


\section{Thema und Fragestellung}

Standardisierte Befragungen stellen heute eine der bedeutendsten Methoden der empirischen Sozialforschung dar; sowohl in der akademischen Forschung, der kommerziellen Forschung als auch in der amtlichen Statistik (Diekmann 20I2). Eine wesentliche methodologische, also auch erkenntnistheoretische Frage ist jedoch, inwiefern verschiedene Erhebungsmodi (survey modes), d.h. Arten der Befragung, die sich potenziell in ihrer spezifischen Befragungssituation und Messqualität unterscheiden, zu ähnlichen substanziellen Ergebnissen führen (Bowling 2005). Hintergrund des Forschungsinteresses an methodischen Themen dieser Art ist insbesondere die zunehmende Verbreitung von für gewöhnlich nicht-probabilistischen oder freiwilligen „opt-in“-Online-Erhebungen mittels CAWI (Computer Assisted Web Interviewing), die eine neue Herausforderung für die quantitativ-empirische Sozialforschung darstellen (für einen Überblick der Diskussion vgl. Baker et al. 20IO).

Unterschiede in den Ergebnissen politischer Meinungsumfragen zwischen Online- und Telefonbefragungen, z.B. hinsichtlich des Ausgangs des Brexit-Votums (z.B. Sayers 20I6) oder der US-Wahl (z.B. Enten 20I6), haben auch zu öffentlichen Diskussionen über die Gültigkeit verschiedener Arten von Umfragen geführt (z.B. auch Aichholzer et al. 2017 für Ergebnisse zur ersten Runde der österreichischen Präsidentschaftswahl im Jahr 20I6). Ein wesentliches Ziel der vorliegenden Studie ist daher, die Ergebnisse aus CAWI-Erhebungen mit jenen traditioneller Methoden - in der vorliegenden Studie konkret CAPI (persönliche, face-to-face Interviews) - zu vergleichen. Hierfür werden Daten der Österreichischen Nationalen Wahlstudie (AUTNES) aus dem Jahr 2013 verwendet, um Erhebungsmodi - CAWI und CAPI - einander gegenüberzustellen. Zentrales Erkenntnisinteresse ist daher generell, inwieweit verschiedene Befragungsmodi ähnliche Ergebnisse liefern.

Fokussiert wird dabei als Fallbeispiel auf berichtetes Wahlverhalten am Beispiel der FPÖ (Freiheitliche Partei Österreichs). Dieses Thema ist für die Wahlforschung von besonderem Interesse, da bekannt ist, dass die berichtete Wahl für rechtspopulistische Parteien wie der FPÖ (d.h. deren geschätzter Anteil) in traditionellen Befragungsmodi oftmals unterschätzt wird bzw. wird deren Anteil in CAWI-Befragungen meist akurater abgebildet (z.B. Aichholzer et al. 2017 zur FPÖ; Bergman/ Diermeier 2017 zur AfD in Deutschland; vgl. auch Sayers 2016 zum Brexit-Votum). Als Folge hätten laut diesen Erkenntnissen Personen in CAWI-Befragungen eine höhere Wahrscheinlichkeit, die FPÖ zu wählen. Wie kann dieser Umstand erklärt werden?

Es wurde vielfach gezeigt, dass die Wählerschaft der FPÖ charakterisiert wird durch jüngere Personen im
Erwerbsalter (vs. in Pension befindliche), Personen mit geringerer formaler Bildung, Politikverdrossene mit geringerer Demokratiezufriedenheit (politische Entfremdung) sowie weitgehend negativen Einstellungen gegenüber Zuwanderung und autoritäre Einstellungen (z.B. Aichholzer et al. 20I4; Aichholzer/Zandonella 20I6; Kritzinger et al. 2013; Lubbers et al. 2002). Eine allgemeine Erklärung für so genannte "Moduseffekte“ (mode effects) könnte lauten, dass sozio-demografische Merkmale, die FPÖ-Wählerinnen und -Wähler charakterisieren, sowie Unterschiede in Einstellungen zu politischen Themen, die mit dem Wahlverhalten korrelieren, mittels CAWI-Stichproben besser erfasst werden können. Im Folgenden wird daher ein einfaches theoretisches Modell vorgestellt. Dieses verknüpft mögliche Hypothesen zu Moduseffekten, um letztlich Unterschiede in der berichteten Parteiwahl bzw. die Scheinkorrelation zwischen Modus und Parteiwahl erklären zu können. Das beschriebene Modell wird schließlich anhand der AUTNES-20I3-Daten empirisch getestet.

Ein erster Erklärungsansatz ist die unterschiedliche sozio-demografische Zusammensetzung der Stichproben. Dazu zählt auch der Umstand, dass weniger Interessierte und Politikverdrossene in CAWI-Befragungen durch "Incentivierung" (üblicherweise finanzielle Anreize) möglicherweise häufiger rekrutiert werden können (Groves et al. 2004). Ein weiterer Erklärungsansatz sind mögliche Unterschiede in der sog. „sozialen Erwünschtheit" des berichteten Wahlverhaltens bzw. jener politischen Einstellungen, welche die Wahl für die FPÖ begünstigen. Soziale Erwünschtheit könnte das Berichten vermeintlich positiv konnotierter Einstellungen im Streben nach sozialer Anerkennung beeinflussen (Paulhus 2002). Somit würde ein „Underreporting“ verschiedener Einstellungen (z.B. Ablehnung von $\mathrm{Zu}$ wanderung oder Musliminnen und Muslimen) bzw. des daraus resultierenden Wahlverhaltens auf Einflüsse sozialer Erwünschtheit im jeweiligen Befragungsmodus zurückzuführen sein. Werden diese Variablen statistisch kontrolliert, sollten folglich direkte Unterschiede in der Wahrscheinlichkeit der Parteiwahl zwischen den Erhebungsmodi „verschwinden“, da lediglich indirekte Einflüsse anzunehmen sind.

Zunächst wird im folgenden Abschnitt das Thema Ursachen von Moduseffekten umrissen, um konkrete Erwartungen für die Analyse abzuleiten. Darauf folgend werden die Daten der AUTNES-Studie beschrieben, die einen Vergleich der Modi CAWI und CAPI erlauben. Im Analyseteil werden die indirekten Effekte des Erhebungsmodus untersucht, um mögliche Mediatorvariablen zu identifizieren, die eine Scheinkorrelation zwischen Modus und Parteiwahl hervorrufen können. Der letzte Abschnitt diskutiert weitere Rückschlüsse aus den Ergebnissen. 


\section{Erklärungsansätze zu Moduseffekten}

Eine breite Literatur hat bislang versucht, sich mit dem Ausmaß und den Ursachen aller Arten von Fehlerquellen in Umfragen auseinanderzusetzen, die als "Total Survey Error" Ansatz zusammengefasst werden kann (Weisberg 2005). Auf der Suche nach einer Erklärung für divergierende Ergebnisse aus unterschiedlichen Erhebungsmodi, also dem sogenannten Moduseffekt, gibt es folgende zentrale Erklärungen: (I.) Selektionseffekte durch spezielle Stichproben und (2.) Messeffekte, wie z.B. systematische Unterschiede im Antwortverhalten oder soziale Erwünschtheit. Diese Aspekte sollen daher im Folgenden beleuchtet werden.

\subsection{Selektionseffekt bzw. Stichprobenunterschiede}

Betrachtet man unterschiedliche Erhebungsmodi, d.h. CAPI, CATI, CAWI oder postalische Befragungen, werden notwendigerweise unterschiedliche Auswahlrahmen für Stichproben (Samples) verwendet, wie Postadressen, Telefonbücher bzw. RDD (Random Digit Dialing) oder die primäre Rekrutierung im Internet. Das erste und offenkundigste Problem der meisten CAWIErhebungen stellt gegenwärtig immer noch die Untererfassung der Zielpopulation (z.B. die wahlberechtigte Bevölkerung) durch die Restriktion auf eine Population mit Internetanschluss dar: bspw. waren nur $85 \%$ aller Privathaushalte in Österreich im Jahr 2016 mit einem Internetzugang ausgestattet, im Jahr 2013 waren es lediglich 81\% (Statistik Austria 20I7). Bekannt ist ebenfalls die sogenannte „digitale Kluft", d.h. demografische und sozioökonomische Unterschiede bzw. Benachteiligung bedingen Unterschiede im Zugang zu Informationsund Kommunikationstechnologien. Eine mögliche Erklärung für unterschiedliche Ergebnisse aus verschiedenen Erhebungsmodi ist daher in Sample-Unterschieden primär sozio-demografischer Natur zu suchen.

Eine gängige Variante, um die Qualität der Stichprobe bzw. Selektionseffekte zu beziffern, ist - sofern möglich -, die Punktschätzer (z.B. Anteile eines Merkmals) aus der Stichprobe mit bekannten Parametern, d.h. einer Benchmark zu vergleichen. Für soziodemografische Merkmale oder faktische Fragen können dies etwa Vergleichszahlen aus offiziellen Registern (amtliche Statistik) sein. Mehrere Studien haben bislang bedeutsame Unterschiede in der demografischen $\mathrm{Zu}$ sammensetzung von Internet-Panels im Vergleich zu probabilistischen Stichproben gefunden, d.h. stärkere Abweichung zu bekannten Populationsparametern (z.B. Dutwin/Buskirk 2017; Pasek 2016; Yeager et al. 201I) sowie stärkere Abweichungen zu bekannten Mustern des Wahlverhaltens (z.B. Malhotra/Krosnick 2007; Sohlberg et al. 20I7).
Das zweite Problem stellt die freiwillige Auswahl bzw. Selbstselektion dar, d.h. Respondentinnen und Respondenten bestimmen ihre Aufnahme in die Stichprobe üblicherweise aufgrund des Themas (z.B. Politik), d.h. dem "topic interest", oder aufgrund finanzieller Anreize (s.g. Incentives), die mögliches Desinteresse kompensieren sollen (Groves et al. 2004). Aus dem Pool der Internetpopulation wird schließlich nach Abzug jener, die an einer bestimmten Erhebung nicht teilnehmen wollen (Unit-Nonresponse), die eigentliche Stichprobe realisiert (Tourangeau et al. 2013). ${ }^{1}$ Es ist jedoch zu erwarten, dass verhältnismäßig mehr politisch Desinteressierte (Personen mit „low topic interest"; siehe Groves et al. 2004) oder Unzufriedene durch die übliche Incentivierung in opt-in-Panels an politischen CAWI-Umfragen teilnehmen. Die Hypothese lautet daher:

Teilnehmerinnen und Teilnehmer in CAWI-Befragungen haben im Schnitt ein geringeres Interesse und eine geringere Anbindung an Politik als jene in CAPIoder CATI-Befragungen, die ohne Incentivierung operieren (Hypothese I).

Man kann außerdem annehmen, dass CAWI-Erhebungen potenziell jene Personengruppen besser erreichen, die in traditionellen Methoden (CATI und CAPI) schlechter erfasst werden: jüngere und mobilere Personen, jene mit geringer formaler Bildung - sofern eine Incentivierung eingesetzt wird - sowie (noch) Erwerbstätige (Hypothese 2).

Warum also finden sich potenziell mehr FPÖ-Wählerinnen und -wähler in CAWI-Stichproben? Die Erklärung läuft darauf hinaus, dass bestimmte Bevölkerungsgruppen in Stichproben von CAWI-Befragungen aufgenommen werden, die in traditionelleren Modi erfahrungsgemäß unterrepräsentiert sind: politisch Desinteressierte, Politikverdrossene, jüngere bzw. mobilere Personen mit geringer formaler Bildung sowie (noch) Erwerbstätige. All diese Faktoren sind jedoch gleichzeitig relevante Prädiktoren der Wahl für die FPÖ (vgl. Aichholzer et al. 20I4; Kritzinger et al. 2013). ${ }^{\text {D Die wahr- }}$ scheinliche Folge ist eine positive Scheinkorrelation zwischen der Teilnahme im Modus CAWI und Wahl der FPÖ (Die Nullhypothese ist selbstverständlich, dass kein solcher Zusammenhang anzunehmen ist).

I Dennoch sollte erwähnt werden, dass andere Erhebungsmethoden, wie z.B. Telefonstichproben, realiter auch nur annährend probabilistisch sind, da über die letzten Jahrzehnte sinkende ResponseRaten in allgemeinen Bevölkerungsumfragen ebenfalls das Problem der Selektion fördern (vgl. Weichbold et al. 2009). Response-Raten mit CAWI sind im Schnitt allerdings immer noch deutlich geringer als in anderen Modi (z.B. CATI und Mail) (Wengrzik et al. 20I6). 


\subsection{Messeffekte und Messqualität: Soziale Erwünschtheit}

Wie bereits erwähnt, stellen unterschiedliche Erhebungsmodi im Prinzip jeweils spezifische Befragungssituationen dar. Wichtige Faktoren können sein: die soziale Situation an sich, wie Beeinflussung durch Anwesenheit von Interviewerinnen oder Interviewern bzw. die Anonymität der Antwort (d.h. im Wesentlichen: CAWI/ paper-pencilvs. CAPI/CATI), die kognitive Belastung für die Respondentinnen und Respondenten sowie die mögliche Kontrolle über die Qualität der Antwort oder die praktische Durchführung der Erhebung (Bowling 2005). All diese Aspekte können letztlich erwünschte oder aber nachteilige Messeffekte auf Antworten in einer Befragung nach sich ziehen, wobei selbstverständlich subjektive Einstellungsfragen (vs. faktische Fragen) anfälliger sind für Moduseffekte (Saris/Gallhofer 2007). ${ }^{2}$

Hier soll allerdings dem Aspekt sozialer Erwünschtheit als Messeffekt besonderes Augenmerk geschenkt werden, da dieser in der Wahlverhaltensforschung eine oft wenig beachtete, jedoch bedeutende Rolle spielen kann. Wahlbeteiligung etwa, gemeinhin als sozial erwünscht erachtet, wird trotz entsprechender Maßnahmen in der Frageformulierung bekanntlich deutlich überschätzt (vgl. Zeglovits/Kritzinger 20I4). Einstellungen zu sensiblen Themen werden als positiver oder wünschenswerter berichtet (Tourangeau/Yan 2007), wie z.B. Haltungen gegenüber Immigration. Hierbei spielt, so die These, der Faktor Fremdtäuschung (Streben nach Gemeinschaft, Teilnahme und Bindung) als „moralistischer" Bias eine Rolle (Paulhus 2002). In Intervieweradministrierten Befragungen bestünde demnach eher eine Tendenz dahingehend als "gute Bürgerin“ oder "guter Bürger" dastehen zu wollen. Die Hypothesen lauten daher:

Personen tendieren aufgrund sozialer Erwünschtheit dazu, ihre Ablehnung gegenüber Zuwanderung oder bestimmten sozialen Gruppen zu "verstecken“ (Hypothese 3a) (Blinder et al. 2013; Janus 2010).

Selbstadministrierte Befragungen, wie CAWI, reduzieren jedoch bekanntermaßen die Effekte von sozialer Erwünschtheit erheblich (Hypothese 3b) (vgl. Bosnjak 20I7; Tourangeau/Yan 2007).

Im konkreten Anwendungsbeispiel politischer Meinungsumfragen und der Wahl der FPÖ spielt soziale Erwünschtheit, so die Hypothese, eine wichtige Rolle. Die These lautet, dass der Anteil rechtspopulistischer Wählerinnen und Wähler, wie jene der FPÖ, in Intervieweradministrierten Modi primär aufgrund von Effekten der

2 Hier nicht betrachtet wird die Messqualität von Einstellungsfragen an sich, wobei Metaanalysen nahe legen, dass Messungen mit CAWI-Interviews sowie Selbstausfüller (Papier) potenziell höhere Messqualität aufweisen, d.h. Messwerte tendenziell höhere Reliabilität und Validität aufweisen (Saris/Gallhofer 2007). sozialen Erwünschtheit unterschätzt wird (vgl. Aichholzer et al. 20I4, 20I7; Bergmann/Diermeier 20I7). Da man soziale Erwünschtheit selten direkt messen kann, ist zunächst anzunehmen, dass insbesondere ablehnende Einstellungen gegenüber Immigrantinnen und Immigranten bzw. Zuwanderung als solche, aber auch rechts-autoritäre Einstellungen im CAWI-Modus offener zugegeben werden. Die Kombination der Hypothesen $3 a$ und $3 b$ ergibt daher:

Erwartet wird ein positiver Zusammenhang zwischen CAWI und der Äußerung von ideologisch rechten bzw. autoritären Einstellungen (allgemeine Hypothese 3).

Die genannten Einstellungsmuster sind allerdings, wie vielfach gezeigt wurde, Kernmerkmal der Wählerschaft der FPÖ (z.B. Aichholzer et al. 20I4; Aichholzer/ Zandonella 2016; Lubbers et al. 2002). Die wahrscheinliche Folge ist daher ebenfalls eine positive Scheinkorrelation zwischen der Teilnahme im Modus CAWI und Wahl der FPÖ (siehe Nullhypothese).

In Summe werden daher folgende Zusammenhänge in einem simplen theoretischen Modell unterstellt (siehe Abbildung I). Die oben genannten Hypothesen werden im Folgenden in Regressionsmodellen über die Bestimmung des Ausmaßes indirekter Effekte getestet (siehe Breen et al. 2013). Das Ziel ist außerdem, mögliche Effekte des Erhebungsmodus „wegzuerklären“, nachdem substanzielle Erklärungsvariablen in das Modell eingeführt worden sind.

Abbildung 1: Theoretisches Modell zur Begründung von Moduseffekten)

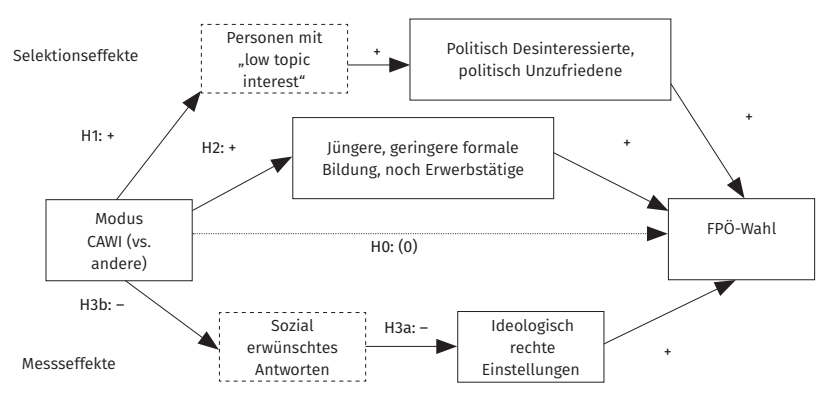

\section{Daten}

Eine Minimalbedingung für den Vergleich der Auswirkungen unterschiedlicher Erhebungsmodi ist letztlich die exakt gleiche Operationalisierung der zu erhebenden Merkmale (Variablen) in den Daten. Um dieses Ziel zu erreichen, ist es notwendig, Daten mit integriertem Design auszuwählen. Zu diesem Zweck wurden Daten aus den separaten Erhebungen der Austrian National Election Study (AUTNES) 2013 untersucht, die rund um 
die Nationalratswahl 2013 erhoben wurden. ${ }^{3}$ Außerdem müssen zur Abschätzung des Moduseffekts Umfragen mit übereinstimmenden Indikatoren (d.h. idente Messung) ausgewählt werden, um deren Einfluss vergleichen zu können. Aus diesem Grund beschränkt sich die vorliegende Studie lediglich auf die im Folgenden beschriebenen Erhebungen (siehe Tabelle I für Details).

\subsection{Verwendete Stichproben}

Die CAWI-Stichprobe basiert auf Daten der „AUTNES Online Panel Study“, die von TNS Opinion (Brüssel) durchgeführt wurde (Kritzinger et al. 20I6a). Die Daten wurden online (CAWI) mittels einer Gelegenheitsstichprobe (Opt-in-Panel) erhoben. Die Feldzeit der Wellen I-4 (Welle 4 = Nachwahl) lief zwischen August bis Oktober 2013. Die Ausgangsstichprobe betrug zu Beginn (Welle I) n = 3084 (I4.8\% Response-Rate bezogen auf kontaktierte Mitglieder des TNS-Online-Panels) und n $=2456$ in der Nachwahlerhebung $(79.6 \%$ Response-Rate zu Welle I). Die Respondentinnen und Respondenten wurden nach bestimmten Quotenmerkmalen kontaktiert (siehe Tabelle I).
(61.8\% Response-Rate) und in der Nachwahlerhebung $\mathrm{n}$ $=1504$ (46.I\% Response-Rate bezogen auf Welle I).

Weitere Details zu den Daten und Stichproben können den entsprechenden Dokumentationen entnommen werden (Kritzinger et al. 20I6a; 20I6b). Der Ergebnisteil bespricht zudem Abweichungen der Stichprobenstrukturen von bekannten Randverteilungen.

\subsection{Variablen in der Analyse}

Eine Liste der vollständig vergleichbaren Variablen und Informationen über deren Codierung findet sich im Appendix (Tabelle AI). Diese lassen sich entsprechend der Hypothesen gliedern in: (a.) soziodemografische Variablen, (b.) Variablen zur Messung politischen Interesses sowie politische Zufriedenheit und (c.) Dimensionen ideologischer Einstellungen. Das heißt, die Aufnahme dieser verschiedenen Variablenblöcke soll klären, weshalb eine mögliche Scheinkorrelation zwischen Erhebungsmodus und Wahl der FPÖ zustande kommt bzw. über welche Variablen eine Mediation des Moduseffekts anzunehmen ist.

Tabelle 1: Datengrundlage (AUTNES-Stichproben)

\begin{tabular}{lccl}
\hline $\begin{array}{c}\text { Komponente } \\
\text { (Modus) }\end{array}$ & $\begin{array}{c}n / R R \\
\text { (Vorwahl) }\end{array}$ & $\begin{array}{c}n / R R \\
\text { (Nachwahl) }\end{array}$ & \multicolumn{1}{c}{ Stichprobe bzw. Auswahlrahmen } \\
\hline 1.) CAWI & 3084 & 2456 & $\begin{array}{l}\text { Quotenrekrutierung aus einem Panel nach: } \\
\text { Geschlecht, Alter, Bundesland, Haushaltsgröße. }\end{array}$ \\
& $14.8 \%$ & $79.6 \%$ & \\
2.) CAPI & 3266 & 1505 & $\begin{array}{l}\text { Postadressen (Zählsprengel), Zufallsauswahl der Person aus } \\
\text { Haushaltsadresse. }\end{array}$ \\
& $61.8 \%$ & $46.1 \%$ & \\
\hline
\end{tabular}

Anmerkung: Für Details siehe die entsprechenden Dokumentationen zu den Datensätzen (Kritzinger et al. 2016a; 2016b); RR = Response-Rate.

Die CAPI-Stichprobe basiert auf Daten des „AUTNES Pre- and Post-Election Survey 2013" (Kritzinger et al. 20I6b). Mit der Durchführung wurde IFES (Institut für empirische Sozialforschung, Wien) beauftragt. Die Daten wurden face-to-face (CAPI) mittels einer mehrfach geschichteten Zufallsstichprobe erhoben, die als Ausgangsbasis statistische Zählsprengel, danach Postadressen und eine Zufallsauswahl der Person aus der Haushaltsadresse vorsah. Die Stichprobe inkludiert zudem ein over-sampling von I6- bis 2I-jährigen Erstwählerinnen und Erstwählern $(\mathrm{n}=200)$. Die Feldzeit für Welle I (Vorwahl) lief zwischen November und Dezember 2012 sowie April bis Juni 20I3. Die Feldzeit für Welle 2 (Nachwahl im CATI-Modus) lief von 30. September bis 2. Dezember. Die Ausgangsstichprobe betrug $n=3266$

3 Alle Daten sind öffentlich über die Seite von GESIS (www.gesis.org) zugänglich.
Danach wurde ein gemeinsamer Datensatz erzeugt (Daten-Matching), der alle vergleichbaren bzw. kongruenten Variablen über die Studien hinweg beinhaltet, um so Unterschiede zwischen den Stichproben/Modi zu bemessen. Dazu wurden alle Fälle der beiden Datensätze zusammengespielt (insgesamt $n=6350$ ). Alle Variablen außer dem berichteten Wahlverhalten (Nachwahl) wurden jeweils in einer Vorwahl-Erhebung gemessen. ${ }^{4}$ Auf eine nachträgliche Gewichtung der Daten wurde in den folgenden Analysen verzichtet. Deren Einfluss auf die Ergebnisse wird jedoch separat im Abschnitt zur Robustheit der Ergebnisse besprochen.

4 Im CAWI-Modus wurde eine Messung für Demokratiezufriedenheit aus Welle 4 (Nachwahl) verwendet. Eine Überprüfung der Analysen mit einer Messung aus Welle 3 (Vorwahl/Kampagne) führt jedoch zu identen substanziellen Ergebnissen. 


\section{Ergebnisse}

\subsection{Selektionseffekte: Soziodemografische Merkmale und Wahlverhalten}

Aus dem Vergleich der ungewichteten Daten der AUTNES-Studien mit den Randverteilungen sechs möglicher Merkmale des Mikrozensus 2012 (Statistik Austria) sowie offiziellen Wahlergebnissen als "Benchmark“ lassen sich die Stichproben zunächst wie folgt beschreiben (siehe Abbildung 2):

Abbildung 2: Abweichungen der demografischen Struktur und des Wahlverhaltens (in \%)

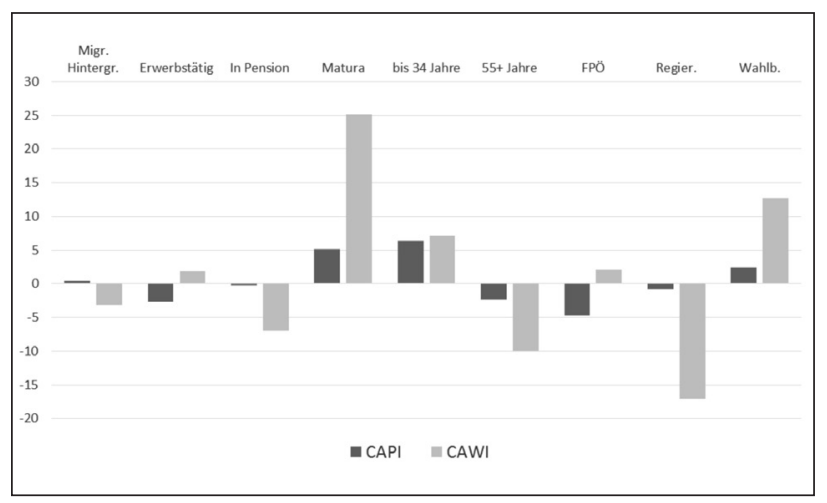

Anmerkung: Die CAPI-Erhebung beinhaltet ein Over-Sampling von 16- bis 21-jährigen Personen. Quellen: Differenz AUTNES-Daten (ungewichtet) zu (a.) Statistik Austria: Mikrozensus 2012 (b.) BM.I - Bundesministerium für Inneres

Insgesamt weist das CAPI-Sample, berücksichtigt man das geplante Over-Sampling junger Menschen (I6- bis 2I-jährige Personen), durchwegs geringere Abweichungen zu bekannten demografischen Charakteristika auf. Das CAWI-Sample weist im Vergleich einen deutlich höheren Anteil an jungen Menschen mit - in diesem Sample - höherer formaler Bildung, jedoch im Gegenzug einen geringeren Anteil an Personen auf, die bereits in Pension oder 55 Jahre und älter sind. Auffallend für beide Erhebungen ist die generelle Überschätzung des formalen Bildungslevels (Anteil mit mindestens Matura) - ein typischer Befund aus sozialwissenschaftlichen Umfragen - die im CAPI-Sample allerdings geringer ausfällt. In Summe führt somit die reine ex-ante Quotierung im CAWI-Sample bei gleichzeitig geringer Response-Rate trotzdem zu deutlichen Abweichungen zu bekannten Populationsparametern.

Neben dem Anteil an FPÖ-Wählerinnen und -Wählern in den beiden Stichproben wurden noch weitere Kennwerte des Wahlverhaltens zu Vergleichszwecken herangezogen. In Bezug auf das Wahlverhalten bei der Nationalratswahl 2013, nämlich (a.) Stimmen für die
FPÖ (offiziell: 20.5\%), (b.) Wahl der Regierungsparteien (SPÖ+ÖVP, offiziell: 50.8\%) und (c.) Wahlbeteiligung (offiziell: $74.9 \%$ ), zeigen sich jeweils ohne Gewichtung bedeutsame Unterschiede im berichteten Wahlverhalten (Abbildung 2): Das CAWI-Sample führt sogar zu einer leichten Überschätzung des Anteils an Personen, die die FPÖ gewählt haben $(+2.2 \%)$, während die CAPI-Stichprobe eine klare Unterschätzung aufweist $(-4.7 \%)$. Im Gegenzug dazu finden sich signifikant weniger Wählerinnen und Wähler der Regierungsparteien (SPÖ+ÖVP) im CAWI-Sample (Unterschätzung -I7.I\%). Außerdem zeigt sich, ebenfalls ungewichtet, eine starke Überschätzung der Wahlbeteiligung, wobei dieses bekannte „Overreporting" im CAPI-Sample weniger drastisch ausfällt $(+2.4 \%$ vs. $+\mathrm{I} 2.7 \%$ für CAWI).

\subsection{Wahl der FPÖ: Erhebungsmodus als erklärende Variable?}

Ziel des nun folgenden multivariaten Modells ist, den offenkundigen Unterschied im Wähleranteil der FPÖ zwischen den Erhebungsmodi CAWI und CAPI zu erklären. Dabei wird versucht, den Effekt des Erhebungsmodus unter Hinzunahme substanziell relevanter Variablen „wegzuerklären“ oder zu begründen. Der direkte Effekt der Variable "Modus“ sollte demnach verschwinden, sofern er primär von anderen Variablen vermittelt (mediiert) wird. Hierfür wird das Verfahren der Effektdekomposition herangezogen, das unter anderem in Strukturgleichungsmodellen angewandt wird, um das Ausmaß der Mediation des Effekts einer Variablen zu beziffern. Die folgenden Analysen für nicht-lineare Modelle (z.B. binär-logistische Regression) stützen sich daher auf der Methode von Breen et al. (2013) zur Dekomposition des totalen Effekts (= direkter Effekt + alle indirekten Effekte) sowie deren Umsetzung mit der Software Stata (Kohler et al. 20II). Der Ansatz erlaubt es entsprechend dem theoretischen Modell, das Ausmaß der Mediation des Effekts einer unabhängigen Variablen (hier: Erhebungsmodus) durch andere Variablen zu bestimmen, d.h. deren indirekter Effekt über mögliche Mediatorvariablen im Verhältnis zum direkten Effekt.

Die Ergebnisse in Tabelle 2 zeigen in der ersten Spalte den totalen Effekt der Variable Modus (CAWI vs. CAPI) auf die Wahl der FPÖ, der mit $b=.650\left(b_{\text {direkt }}+b_{\text {indirekt }}=\right.$ $.205+.445)$ hoch signifikant ausfällt $(p<.00 I)$. Dieser Effekt bestätigt die erwartete Scheinkorrelation zwischen Befragungsmodus (CAWI) und Parteiwahl, d.h. eine höhere Wahrscheinlichkeit für Personen in der CAWIBefragung die FPÖ zu wählen. Allerdings basiert der Koeffizient auf einem reduzierten Modell ohne Kontrolle von Einflüssen durch Drittvariablen (Konfundierung). Die Spalte zu direkten Effekten legt hingegen nahe, dass de facto kein signifikanter direkter Effekt zwischen Befragungsmodus und Parteiwahl besteht $\left(b_{\text {direkt }}=.205, p=\right.$ 
Tabelle 2: Binär-logistische Regression mit Effektdekomposition (abh. Var.: Wahl der FPÖ)

\begin{tabular}{|c|c|c|c|c|c|c|c|c|}
\hline Effekt & Total & S.E. & Direkt & S.E. & $\begin{array}{r}\text { Indirekt } \\
\text { (für Modus) }\end{array}$ & S.E. & $\begin{array}{l}\% \text { Mediation d. } \\
\text { totalen Effekts }\end{array}$ & $\begin{array}{r}\text { Zus. mit } \\
\text { Modus }\end{array}$ \\
\hline Modus (CAWI = 1) & $.650^{\star \star *}$ & .133 & .205 & .143 & $.445^{\star \star \star}$ & .084 & Summe: 68.5 & \\
\hline Geschlecht & & & -.086 & .131 & .003 & .004 & 0.4 & \\
\hline Matura & & & $-.556^{\star \star *}$ & .140 & $-.089 * * *$ & .025 & -13.7 & - \\
\hline Alter & & & $-.018^{* *}$ & .006 & $.069 * *$ & .026 & 10.6 & - \\
\hline Angestellte/r & & & .080 & .148 & .009 & .016 & 1.3 & \\
\hline Arbeiter/in & & & $.393^{*}$ & .210 & -.046 & .025 & -7.0 & - \\
\hline In Pension & & & -.251 & .206 & .020 & .017 & 3.1 & \\
\hline Schüler/Studierende & & & -.591 & .295 & -0.16 & .010 & -2.5 & \\
\hline Sonstige Erwerbstätigkeit & & & .074 & .202 & .002 & .005 & 0.3 & \\
\hline Negative Einkommenssituation & & & $.416^{\star *}$ & .133 & $.035^{*}$ & .014 & 5.5 & + \\
\hline Migrationshintergrund & & & .064 & .309 & -.001 & .003 & -0.1 & \\
\hline Gewerkschaftsmitglied & & & $-.343^{*}$ & .155 & .000 & .006 & 0.0 & \\
\hline kleine/mittlere Stadt & & & -.233 & .153 & -.001 & .004 & -0.2 & \\
\hline Großstadt Zentrum/Rand & & & .080 & .148 & .004 & .008 & 0.6 & \\
\hline \multirow[t]{2}{*}{ Subjektive Religiosität (Dummy) } & & & -.219 & .128 & .012 & .008 & 1.8 & \\
\hline & & & & & & \multicolumn{3}{|c|}{ Summe: 0.0} \\
\hline Negative Wirtschaftslage & & & .033 & .068 & .006 & .012 & 0.9 & \\
\hline Demokratiezufriedenheit & & & $-.541^{* \star *}$ & .082 & $.177^{\star \star *}$ & .032 & 27.2 & - \\
\hline \multirow[t]{2}{*}{ Politisches Interesse } & & & .001 & .080 & .000 & .011 & 0.0 & \\
\hline & & & & & & \multicolumn{3}{|c|}{ Summe: 28.1} \\
\hline Ablehn. Einstellung bzgl. Zuwanderung & & & $.455^{\star \star *}$ & .086 & $.097^{* *}$ & .029 & 15.0 & + \\
\hline Ablehn. Einstellung bzgl. Muslimen & & & $.359^{* * *}$ & .086 & $.078^{* *}$ & .025 & 12.1 & + \\
\hline Links-Rechts-Skala & & & $.267^{\star * *}$ & .035 & $.049^{*}$ & .023 & 7.5 & + \\
\hline \multirow[t]{2}{*}{ Autoritäre Einstellungen } & & & $.329 * *$ & .120 & $.038^{*}$ & .017 & 5.8 & + \\
\hline & & & & & & \multicolumn{3}{|c|}{ Summe: 40.4} \\
\hline$n$ & & & 2470 & & & & & \\
\hline McFadden Pseudo- $R^{2}$ & & & .30 & & & & & \\
\hline
\end{tabular}

Anmerkung: Effektdekomposition nach Breen et al. (2013) sowie Kohler et al. (2011). Informationen zur Variablencodierung finden sich im Appendix. Einträge zeigen Logit-Koeffizienten, S.E. = Standardfehler. Zweiseitige Signifikanz: * $p<.05,{ }^{* *} p<.01,{ }^{* * *} p<.001$

.152). Die Nullhypothese sollte somit beibehalten werden bzw. die Variable selbst hat keine weitere Erklärungskraft. Weiters zeigt die Spalte der direkten Effekte, wie gewohnt, die für die Forscherin oder den Forscher relevanten substanziellen Einflüsse der untersuchten Variablen auf die Wahl der FPÖ (vs. andere Wahloptionen). Diese bestätigen weitgehend bereits bekannte Determinanten der Wahl rechtspopulistischer Parteien (z.B. Aichholzer et al. 2014; Lubbers et al. 2002).

\subsection{Mediationseffekte: Zerlegung indirekter Effekte des Erhebungsmodus}

Im Rahmen des hier behandelten Themas ist nun die jeweilige Mediation des Effekts des Befragungsmodus über andere Variablen von Interesse, die in Tabelle 2 in der Spalte indirekter Effekte dargestellt werden. Deren Summe $\left(b_{\text {indirekt }}=.445, p<\right.$.OOI $)$ lässt sich auch als Anteil 
Tabelle 3: OLS-Regressionen für potenzielle Mediatorvariablen

\begin{tabular}{|c|c|c|c|c|c|c|c|c|}
\hline \multirow{2}{*}{$\begin{array}{l}\text { Abhängige Variable } \\
\text { Effekt }\end{array}$} & \multicolumn{2}{|c|}{$\begin{array}{l}\text { Demokratie- } \\
\text { zufriedenheit } \\
\left(\mathrm{W}_{4} / \text { Nachwahl }\right)\end{array}$} & \multicolumn{2}{|c|}{$\begin{array}{l}\text { Demokratie- } \\
\text { zufriedenheit } \\
\left(\mathrm{W}_{3} / \text { Vorwahl }\right)\end{array}$} & \multicolumn{2}{|c|}{$\begin{array}{l}\text { Ablehnende } \\
\text { Einstellung bzgl. } \\
\text { Muslimen }\end{array}$} & \multicolumn{2}{|c|}{$\begin{array}{c}\text { Autoritäre } \\
\text { Einstellungen }\end{array}$} \\
\hline & b & S.E. & b & S.E. & b & S.E. & b & S.E. \\
\hline Modus (CAWI = 1) & $-.210^{\star \star \star}$ & .024 & $-.142^{\star \star \star}$ & .028 & $.087^{\star \star \star}$ & .025 & $.110^{\star \star \star}$ & .018 \\
\hline \multicolumn{9}{|c|}{$\begin{array}{l}\text { Zusätzlich sind alle Variablen } \\
\text { aus Tabelle } 2 \text { inkludiert }\end{array}$} \\
\hline$n$ & 4220 & & 3503 & & 4220 & & 4220 & \\
\hline Adj. $R^{2}$ (volles Modell) & .17 & & .17 & & .56 & & .32 & \\
\hline Adj. $R^{2}$ (ohne Modus) & .16 & & .16 & & .56 & & .32 & \\
\hline
\end{tabular}

Anmerkung: Volle Stichprobe aus Vorwahl. Informationen zur Variablencodierung finden sich im Appendix. Einträge zeigen unstandardisierte Regressionskoeffizienten, S.E. = Standardfehler. Zweiseitige Signifikanz: ${ }^{*} p<.05,{ }^{* *} p<.01,{ }^{* * *} p<.001$

am totalen Effekt ausdrücken (vgl. Breen et al. 2013). Der Großteil (68.5\%) des Einflusses des Befragungsmodus auf das Wahlverhalten wird demzufolge in Wahrheit durch andere Variablen - d.h. jene, die im Modell inkludiert werden konnten - mediiert. Die Spalte „\% Mediation" zeigt somit den Anteil des ursprünglichen Effekts des Erhebungsmodus in einem reduzierten Modell, der de facto durch die jeweilige Variable konfundiert (d.h. verunreinigt) wird. Auch lässt sich das Ausmaß der Mediation nach Variablengruppen aufschlüsseln: Demzufolge tragen sozio-demografische Variablen in Summe kaum oder gar nicht zur Scheinkorrelation zwischen Erhebungsmodus (CAWI) und Wahl der FPÖ bei (0.0\% Mediation des Effekts), hingegen tun dies speziell der Faktor geringe Demokratiezufriedenheit (27.2\% Mediation des Effekts) und zu einem noch bedeutenderen Teil (40.4\% Mediation des Effekts) die Summe aller ideologischen Einstellungen.

Für statistisch signifikante Variablen wurde außerdem deren Zusammenhang mit dem Modus (CAWI) verdeutlicht. Aus dem direkten Effekt der jeweiligen Variablen und dem indirekten Effekt des Befragungsmodus, der über diese Variable läuft, lässt sich damit rückschließen, welche Gruppen im Modus CAWI unter- oder überrepräsentiert sind (letzte Spalte in Tabelle 2, „Zus. mit Modus CAWI“). Der negative direkte Effekt für Matura und der indirekte Effekt für CAWI bedeutet für diese Daten, dass höher Gebildete und Jüngere im Modus CAWI tendenziell überrepräsentiert waren, Arbeiterinnen und Arbeiter unterrepräsentiert, Personen mit negativer Einkommenssituation überrepräsentiert, politisch Zufriedene unterrepräsentiert und dass ideologisch rechts orientierte Personen überrepräsentiert waren bzw. wurden entsprechende Einstellungen häufiger berichtet - jeweils unter Konstanthaltung der anderen Variablen im Modell.
Um die genannten Zusammenhänge zu verdeutlichen, wurden zusätzlich für ausgewählte Mediatorvariablen einfache OLS-Regressionen mit dem vollen Sample (Vorwahl) berechnet. 5 Der Erhebungsmodus fungiert wiederum als erklärende Variable (siehe Tabelle 3), jedoch wird gleichzeitig für eine Vielzahl substanzieller Erklärungsvariablen kontrolliert. ${ }^{6}$ Im Unterschied zur Parteiwahl bleibt dabei in allen angeführten Beispielen eine (hoch) signifikante Resterklärungskraft bestehen, wenngleich der Gewinn an erklärter Varianz $\left(R^{2}\right)$ gering ausfällt. Die Ergebnisse besagen daher bspw. dass: Personen im Modus CAWI (im Vergleich zu CAPI) häufiger Unzufriedenheit mit der Demokratie in Österreich äußern, stärker ablehnende Einstellungen bzgl. Musliminnen und Muslimen äußern sowie deutlich autoritärere Einstellungen aufweisen.

\subsection{Robustheit der Ergebnisse: Auswirkung einer Poststratifikationsgewichtung}

In der Literatur gibt es eine Kontroverse, ob eine zusätzliche Gewichtung der Daten in Regressionsmodellen sinnvoll ist, sofern die Gewichte aus Merkmalen erstellt wurden, die auch Teil der Regressionsgleichung sind (vgl. Winship/Radbill I994). Aus diesem Grund soll die Robustheit der Ergebnisse bei Hinzunahme einer Gewichtung untersucht werden. Das Gewichtungsverfahren, das in AUTNES verwendet wurde, wird auch als Zellgewichtung oder als Poststratifikationsgewichtung (post-stratification) bezeichnet, wobei die Randvertei-

5 Dies inkludiert Personen, die schließlich nicht mehr erreicht wurden oder nicht an der Wahl teilgenommen haben.

6 Demokratiezufriedenheit wurde als ordinale Variable gemessen, hingegen in der Analyse als metrisch angenommen. Die Ergebnisse aus einem ordinal-logistischen Modell sind jedoch inhaltlich ident. 
lungen der Stichprobe in einem iterativen Verfahren an bekannte Randverteilungen der Population angepasst werden. Gewichtet wurde in AUTNES nach den Ergebnissen des Mikrozensus 2012 (Statistik Austria), wobei folgende Merkmale bzw. deren Kombination verwendet wurden: Geschlecht, Alter, Bildung, Migrationshintergrund, Bundesland, Haushaltsgröße, Erwerbsstatus, Geschlecht x Matura und Alter (kategorisiert) x Matura.

Die Auswirkung einer Gewichtung (hier: Nachwahlstichprobe) zeigt, dass der direkte Effekt des Erhebungsmodus auf das Wahlverhalten noch geringer ausfällt $\left(b_{\mathrm{di}-}\right.$ rekt $=.064, p=.76 \mathrm{I}$ vs. $b_{\text {indirekt }}=.8 \mathrm{II}, p<$.00I) und der überwiegende Teil des Einflusses auf die Parteiwahl somit mediiert wird (92.6\%). Die Mediation über ideologische Variablen tritt jedoch noch deutlicher hervor $(65.4 \% \mathrm{Me}-$ diation des Effekts), ebenso die Mediation über geringe Demokratiezufriedenheit (21.8\%), wohingegen soziodemografische Unterschiede weiterhin wenig zur Erklärung von Mediationseffekten beitragen (5.6\%). Auch bleibt die erwähnte Resterklärungskraft des Modus für die in Tabelle 3 genannten Mediatorvariablen (jeweils bei $p<$.ooI) nach einer Gewichtung bestehen.

\section{Diskussion}

\subsection{Zusammenfassung und Rückschlüsse}

Für den Großteil der Kennwerte soziodemografischer Merkmale sowie des Wahlverhaltens (Stimmenanteile, Wahlbeteiligung) gilt, dass sich die CAPI-Erhebung aus einer Zufallsstichprobe mit Postadressen als überlegen erweist. Nicht zuletzt ein Bericht der AAPOR (American Association for Public Opinion Research) weist deshalb darauf hin, dass reine opt-in-CAWI-Panels derzeit nicht für akkurate Punktschätzer von Bevölkerungsmerkmalen geeignet sind (Baker et al. 20IO). Dieselbe Einschränkung für reine online-Erhebungen wurde bereits für die sogenannte „Sonntagsfrage” in Wahlumfragen geäußert (z.B. VdMI 20I7).

Im vorliegenden Beitrag wurde darüber hinaus versucht, der Frage nach Moduseffekten über die Untersuchung von indirekten Effekten auf eine Zielvariable, nämlich Wahlverhalten, durch mögliche Mediatorvariablen nachzugehen. Den Ergebnissen zufolge tragen Unterschiede in der sozio-demografischen Zusammensetzung kaum zur Konfundierung im Zusammenhang zwischen Erhebungsmodus (CAWI) und Wahl der FPÖ bei (siehe Hypothese 2). Hingegen sind geringe Demokratiezufriedenheit (politisch Entfremdete) und ideologisch rechts gerichtete Einstellungen (z.B. Ablehnung von Immigration sowie autoritäre Einstellungen), beides starke Determinanten der Wahl rechtspopulistischer Parteien (Aichholzer et al. 2014; Aichholzer/Zandonella 2016;
Lubbers et al. 2002), unter Personen im Befragungsmodus CAWI überrepräsentiert bzw. werden häufiger berichtet (siehe Hypothese I und Hypothese 3). Als Folge dürfte es zur bereits erwähnten Scheinkorrelation zwischen Erhebungsmodus und Parteiwahl kommen. Wichtig zu betonen ist, dass es somit nach Kontrolle der im Modell enthaltenen soziodemografischen und politischen Variablen de facto keine Unterschiede in der vorhergesagten Wahrscheinlichkeit der Wahl der FPÖ zwischen den Erhebungsmodi gibt.

Zur Hypothese über die vermehrte Rekrutierung von Personen mit "low topic interest" in CAWI-Umfragen lässt sich sagen, dass zwar politisches Interesse per se keine Rolle zu spielen scheint, allerdings politisch Entfremdete und Unzufriedene mittels CAWI häufiger erreicht wurden (siehe Hypothese I). Zur Hypothese der Effekte geringerer sozialer Erwünschtheit ideologisch rechter Einstellungen lässt sich sagen, dass dieser Faktor offenbar eine bedeutendere Rolle für Unterschiede zwischen den Erhebungsmodi spielt. Auch nach Berücksichtigung anderer Prädiktoren, d.h. substantieller Variablen, werden solche Einstellungen häufiger/stärker im anonymen CAWI-Modus im Vergleich zum persönlich administrierten CAPI-Modus geäußert (siehe Hypothese 3). Wie kann dieser Umstand theoretisch erklärt werden?

Wie erwähnt, gilt Fremdtäuschung gemeinhin als zentrale Motivation sozial erwünschter Antworten in Umfragen (d.h. Streben nach Gemeinschaft, Teilnahme und Bindung, vgl. Paulhus 2002). Nun argumentieren bspw. Blinder et al. (2013), dass in den meisten westlichen Demokratien Normen gegen Vorurteile und Diskriminierung von Minderheiten und insbesondere offenen Rassismus vorherrschen. Starke Ablehnung oder Vorurteile gegenüber dem Zusammenleben mit Ausländerinnen und Ausländern, aber auch rechts-autoritäre Einstellungen, die stark mit der Abwertung von OutGroups und Vorurteilen verwoben sind (vgl. Aichholzer/ Zeglovits 2015), würden demzufolge als tendenziell sozial unerwünscht gelten. Allerdings ist es selbstverständlich, dass Normen, und damit soziale (Un)Erwünschtheit (gesellschafts-)politischer Positionen keine gesellschaftliche Konstante sind. Das öffentliche Meinungsklima kann sich bspw. in Folge der Legitimierung durch neue Parteipositionen wandeln und bislang normativ sanktionierte Meinungen werden "salonfähig" (vgl. Blinder et al. 20I3). Verschiebungen dieser Art, etwa als Folge der sogenannten europäischen „Flüchtlingskrise“ im Jahr 2015, sind dafür sicher ein treffendes Beispiel. Weitere Forschung könnte daher untersuchen, ob sich Trends dieser Art, d.h. solche Verschiebungen des Meinungsklimas in Bezug auf anerkannte Normen im öffentlichen Diskurs, empirisch nachzeichnen lassen. 


\subsection{Ausblick}

Wie kann nun dem sogenannten Moduseffekt begegnet werden? Ein Ziel könnte sein, die Untererfassung bestimmter Bevölkerungsgruppen in traditionellen Modi (CAPI, aber auch CATI) bereits im Vorfeld auszugleichen, d.h. disproportional mehr Personen aus bekanntermaßen unterrepräsentierten Gruppen zu kontaktieren. Dies kann sich allerdings in der Praxis als aufwendig oder auch unmöglich erweisen. Auch lassen sich damit nicht systematische Exklusion oder die berichteten Messeffekte kompensieren. Eine Alternative ist, wie vielfach vorgeschlagen, verschiedene Erhebungsmodi zu mischen (s.g. Mixed-Mode Surveys), da es jeweils nachteilige, aber auch wünschenswerte Effekte bestimmter Erhebungsmodi geben kann (Bosnjak 20I7; De Leeuw 2005; Krug et al. 2017; zu politischen Umfragen z.B. Aichholzer et al. 2017). Ziel der Mischung von Erhebungsmodi ist daher im Grunde, mögliche Selektionseffekte und Messeffekte der spezifischen Erhebungsmodi in Summe auszubalancieren. Dies lässt jedoch die Frage weitgehend offen, wie eine „optimale“ Kombination konkret aussehen könnte, wozu es bislang kaum bis keine Forschung gibt (Bosnjak 2017). Parallel dazu gibt es seit einiger Zeit Bestrebungen in Europa (vgl. Blom et al. 2016), und mit dem PUMA-Projekt neuerdings auch in Österreich, „echte“ probabilistische Online-Panels aufzubauen, die gar nicht oder weniger von Selektionseffekten beeinträchtigt werden.? Im Gegensatz zu üblichen Gelegenheitsstichproben, wird der Stichprobenrahmen im Zuge der Rekrutierung bestmöglich auf die angestrebte Zielpopulation erweitert, um so eine echte Zufallsstichprobe zu realisieren. Ein zusätzlicher, nicht zu verachtender Grund für die Ausweitung der Verwendung von CAWI-Befragungen, ergänzend zu oder statt traditionellen Methoden, ist letztlich die deutliche Reduktion von Kosten in der Feldarbeit. Weitere Forschung sollte daher bspw. den bestmöglichen Einsatz von Incentivierungen untersuchen, um die Kosten-Nutzen-Balance von CAWI-Befragungen sicherzustellen.

\subsection{Einschränkungen der Ergebnisse}

Als Einschränkung der Ergebnisse ist zu erwähnen, dass die hier verwendeten Umfragen der AUTNES 2013 nicht als exaktes "head-to-head"-Design angelegt wurden, wobei die Zuweisung zu einem Modus vollständig randomisiert wird (vgl. dazu Bosnjak 20I7). Im Idealfall müssten hierfür Stichproben zufällig auf Modi verteilt sein, d.h. eine gleichzeitige Randomisierung der Messsituation, und weitgehend idente Response-Raten mit ähnlichen Stichprobenverzerrungen aufweisen, um den Moduseffekt exakter zu bemessen. Dies ist jedoch

7 Siehe:www.puma-plattform.at/puma-umfragen[Zugriff: 12.09.2018] in der Praxis schwer zu bewerkstelligen (Bosnjak 2017; De Leeuw 2005). Die zentrale Herausforderung in der Untersuchung von Effekten durch die Verwendung unterschiedlicher Erhebungsmodi bleibt daher, Selektionseffekte (Stichprobenunterschiede durch Selektion bzw. Non-Response) und Messeffekte (Unterschiede in der Messqualität und -situation) korrekt zu trennen (vgl. De Leeuw 2005; Voogt/Saris 2005). Außerdem wurden im CAPI-Sample zwar alle sozio-demografischen und Einstellungsvariablen mittels CAPI erhoben, das Wahlverhalten aber schließlich in der Nachwahlbefragung im CATI-Modus abgefragt. Da jedoch beide Modi Interviewer-administriert sind, sollte dies die Rückschlüsse kaum beeinflussen.

Eine weitere Einschränkung ist, dass die wahrgenommene soziale Erwünschtheit von politischen Einstellungen in der jeweiligen Messsituation nicht direkt gemessen wurde, sondern nur indirekt darauf rückgeschlossen wurde. Andere Forschungsdesigns, eventuell experimenteller Natur, könnten diesen Faktor exakter untersuchen. Zusammenfassend gesprochen wären daher weitere Analysen zu den hier präsentierten Ergebnissen über indirekte Moduseffekte auf berichtete Einstellungen und Verhalten in politischen Umfragen wünschenswert.

\section{Literatur}

Aichholzer, Julian/Nikolaus Eder/Eva Zeglovits (2017), „The pollsters got it wrong, again?" Combining modes in electoral research. 7 th Conference of the European Survey Research Association (ESRA), Lissabon, Juli 20I7, URL: https://www.researchgate.net/publication/31942276I_Combining_modes_in_electoral_ research [Zugriff: I2.09.2018].

Aichholzer, Julian/Sylvia Kritzinger/Markus Wagner/Eva Zeglovits (2014), How has radical right support transformed established political conflicts? The case of Austria, in: West European Politics, Vol. 37, II3-I37.

Aichholzer, Julian/Martina Zandonella (2016), Psychological bases of support for radical right parties, in: Personality and Individual Differences, Vol. 96, I85-190.

Aichholzer, Julian/Eva Zeglovits (2015), Balancierte Kurzskala autoritärer Einstellungen (B-RWA-6), in: Zusammenstellung sozialwissenschaftlicher Items und Skalen, URL: http://zis.gesis.org/pdfFiles/ Dokumentation/Aichholzer+\%2oBalancierte\%20 Kurzskala\%2oautoritaerer\%2oEinstellungen\%20 (B-RWA-6).pdf [Zugriff: I2.09.2018].

Ansolabehere, Stephen/Brian F. Schaffner (2014), Does survey mode still matter? Findings from a 2010 multi-mode comparison, in: Political Analysis, Vol. 22, 285-303. 
Baker, Reg/Stephen J. Blumberg/J. Michael Brick/Mick P. Couper/Melanie Courtright/J. Michael Dennis/Don Dillman/Martin R. Frankel/Philip Garland/Robert M. Groves/Courtney Kennedy/Jon A. Krosnick/ Paul J. Lavrakas/Sunghee Lee/Michael Link/Linda Piekarski/ Kumar Rao/Randall K. Thomas/Dan Zahs (20IO), Research synthesis: AAPOR report on online panels, in: Public Opinion Quarterly, Vol. 74, 7II-78I.

Bergmann, Knut/Matthias Diermeier (2017), Die AfD: Eine unterschätzte Partei. Soziale Erwünschtheit als Erklärung für fehlerhafte Prognosen, in: IW-Report, Vol. 7/20I7, I-I6.

Blinder, Scott/Robert Ford/Elisabeth Ivarsflaten (2OI3), The better angels of our nature: How the antiprejudice norm affects policy and party preferences in Great Britain and Germany, in: American Journal of Political Science, Vol. 57, 84I-857.

Blom, Annelies G./Michael Bosnjak/Anne Cornilleau/ Anne-Sophie Cousteaux/Marcel Das/Salima Douhou/ Ulrich Krieger (2016), A comparison of four probability-based online and mixed-mode panels in Europe, in: Social Science Computer Review, Vol. 34, 8-25.

Bosnjak, Michael (2017), Mixed-Mode Surveys and Data Quality: Meta-Analytic Evidence and Avenues for Future Research, in: Eifler, Stefanie/Frank Faulbaum (Hg.), Methodische Probleme von Mixed-Mode-Ansätzen in der Umfrageforschung, Wiesbaden: VS Verlag, II-25.

Bowling, Ann (2005), Mode of questionnaire administration can have serious effects on data quality, in: Journal of Public Health, Vol. 27, 28I-29I.

Breen, Richard/Kristian B. Karlson/Anders Holm (2OI3), Total, direct, and indirect effects in logit and probit models, in: Sociological Methods \& Research, Vol. 42, I64-I9I.

De Leeuw, Edith D. (2005), To mix or not to mix data collection modes in surveys, in: Journal of Official Statistics, Vol. 2I, 233-255.

Diekmann, Andreas (2012), Empirische Sozialforschung: Grundlagen, Methoden, Anwendungen (6. Aufl.), Reinbek: Rowohlt.

Dutwin, David/Trent D. Buskirk (2017), Apples to oranges or gala versus golden delicious? Comparing data quality of nonprobability Internet samples to low response rate probability samples, in: Public Opinion Quarterly, Vol. 8I(SI), 213-239.

Enten, Harry (2016), Live Polls And Online Polls Tell Different Stories About The Election, URL: https:// fivethirtyeight.com/features/live-polls-and-onlinepolls-tell-different-stories-about-the-election [Zugriff: 12.09.2018].

Groves, Robert M./Stanley Presser/Sarah Dipko (2004), The role of topic interest in survey participation decisions, in: Public Opinion Quarterly, Vol. 68, 2-3I.
Janus, Alexander L. (2010), The influence of social desirability pressures on expressed immigration attitudes, in: Social Science Quarterly, Vol. 9I, 928-946.

Kohler, Ulrich/Kristian B. Karlson/Anders Holm (2OII), Comparing coefficients of nested nonlinear probability models using khb, in: Stata Journal, Vol. II, $420-438$.

Kritzinger, Sylvia/David Johann/Kathrin Thomas/Christian Glantschnigg/Julian Aichholzer/Konstantin Glinitzer/Johann Gründl/Patricia Oberluggauer/Markus Wagner (20I6a), AUTNES Online Panel Study, Köln: GESIS Datenarchiv (ZA6594, Version I.o.o).

Kritzinger, Sylvia/Eva Zeglovits/Julian Aichholzer/Christian Glantschnigg/Konstantin Glinitzer/David Johann/ Kathrin Thomas/Markus Wagner (2016b), AUTNES Pre- and Post-Election Survey 2013, Köln: GESIS Datenarchiv (ZA5859, Version 2.0.0).

Kritzinger, Sylvia/Eva Zeglovits/Michael S. Lewis-Beck/ Richard Nadeau (2013). The Austrian Voter, Wien: V\&R unipress.

Krug, Gerhard/Johann Carstensen/Peter Kriwy (2017), Die richtige Mischung? Ein randomisiertes Experiment zur Datenqualität bei der Kombination von Telefonund Onlineerhebung in der empirischen Sozialforschung, in: Zeitschrift für Soziologie, Vol. 46, 89-Io6.

Lubbers, Marcel/Mérove Gijsberts/Peer Scheepers (2002), Extreme right-wing voting in Western Europe, in: European Journal of Political Research, Vol. 4I, 345-378.

Malhotra, Neil/Jon A. Krosnick (2007), The effect of survey mode and sampling on inferences about politi$\mathrm{cal}$ attitudes and behavior: Comparing the 2000 and 2004 ANES to Internet surveys with nonprobability samples, in: Political Analysis, Vol. 15, 286-323.

Pasek, Josh (2016), When will nonprobability surveys mirror probability surveys? Considering types of inference and weighting strategies as criteria for correspondence, in: International Journal of Public Opinion Research, Vol. 28, 269-29I.

Paulhus, Delroy L. (2002), Socially desirable responding: The evolution of a construct, in: Braun, Henry I./Douglas N. Jackson/David E. Wiley (Hg.), The role of constructs in psychological and educational measurement, Mahwah, NJ: Erlbaum, 49-69.

Saris, Willem E./Irmtraud N. Gallhofer (2007), Design evaluation and analysis of questionnaires for survey research, Hoboken, NJ: Wiley.

Sayers, Freddie (2016), The online Polls were RIGHT, and other lessons from the referendum, URL: https:// yougov.co.uk/news/2016/06/28/online-polls-wereright [Zugriff: I2.09.2018].

Sohlberg, Jacob/Mikael Gilljam/Johan Martinsson (2017), Determinants of polling accuracy: the effect of opt-in Internet surveys, in: Journal of Elections, Public Opinion and Parties, DOI: IO.IO80/17457289.2017.1300588. 
Statistik Austria (2017), Haushalte mit Internetzugang 2002 bis 20I7, URL: https://www.statistik.at/web_de/ statistiken/energie_umwelt_innovation_mobilitaet/ informationsgesellschaft/ikt-einsatz_in_haushalten/O4IOI5.html [Zugriff: I2.09.20I8].

Tourangeau, Roger/Frederick G. Conrad/Mick P. Couper (2013), The science of web surveys, Oxford: Oxford University Press.

Tourangeau, Roger/Ting Yan (2007), Sensitive questions in surveys, in: Psychological Bulletin, Vol. I33, 859-883.

VdMI - Verband der Markt- und Meinungsforschungsinstitute Österreichs (20I7), Richtlinie für die Erstellung und Veröffentlichung von Ergebnissen der Wahlforschung - sogenannte "Sonntagsfrage" - in Medien für alle Wahlgänge, Url: http://vdmi.at/ wp-content/uploads/2017/o2/Richtlinie_Wahlforschung_oI-20I7.pdf [Zugriff: I2.09.20I8].

Voogt, Robert J.J./Willem E. Saris (2005), Mixed mode designs: Finding the balance between nonresponse bias and mode effects, in: Journal of Official Statistics, Vol. 2 I, 367-387.

Weichbold, Martin/Johann Bacher/Christof Wolf (Hg.) (2009), Umfrageforschung. Herausforderungen und Grenzen (Österreichische Zeitschrift für Soziologie, Sonderheft 9), Wiesbaden: VS Verlag.

Weisberg, Herbert F. (2005), The Total Survey Error Approach: A Guide to the New Science of Survey Research. Chicago, London: The University of Chicago Press.

Wengrzik, Jessica/Michael Bosnjak/Katja Lozar Manfreda (2016), Web Surveys versus Other Survey Modes - A Meta-Analysis Comparing Response Rates, General Online Research (GOR) Conference, März 20I6, Dresden, URL: https://www.researchgate.net/publication/303952684_Web_Surveys_versus_Other_ Survey_Modes_-_A_Meta-Analysis_Comparing_ Response_Rates [Zugriff: OI.09.20I7].

Winship, Christopher/Larry Radbill (1994), Sampling weights and regression analysis, in: Sociological Methods \& Research, Vol. 23, 230-257.

Yeager, David S./Jon A. Krosnick/LinChiat Chang/Harold S. Javitz/Matthew S. Levendusky/Alberto Simpser/Rui Wang (2OII), Comparing the accuracy of RDD telephone surveys and internet surveys conducted with probability and non-probability samples, in: Public Opinion Quarterly, Vol. 75, 709-747.

Zeglovits, Eva/Sylvia Kritzinger (2014), New attempts to reduce overreporting of voter turnout and their effects, in: International Journal of Public Opinion Research, Vol. 26, 224-234.

\section{Autor}

Julian Aichholzer ist Universitätsassistent (Post-Doc) am Institut für Staatswissenschaft der Universität Wien und ist mit dem Vienna Center for Electoral Research (VieCER), der Austrian National Election Study (AUTNES) sowie dem Forschungsverbund „Interdisziplinäre Werteforschung " assoziiert. Seine Forschungsinteressen liegen im Bereich politischer Einstellungen und Wahlverhalten, politischer Psychologie sowie Fragen der Messung in Umfragen. 


\section{Anhang}

Tabelle A1: Codierung der Variablen im kombinierten Datensatz

\begin{tabular}{|c|c|c|}
\hline Variable & Messung & Codierung \\
\hline Parteiwahl (FPÖ) & $\begin{array}{l}\text { Nachwahl } \\
\text { Moduswechsel } \\
\text { CAPI-CATI) }\end{array}$ & 1 = Wahl Partei; 0 = alle anderen Antworten; weiß nicht $/$ k.A. = missing. \\
\hline Modus & Vorwahl & $1=\mathrm{CAWI}, 0=\mathrm{CAPI}$. \\
\hline Geschlecht & Vorwahl & 1 = männlich, 0 = weiblich. \\
\hline Matura & Vorwahl & 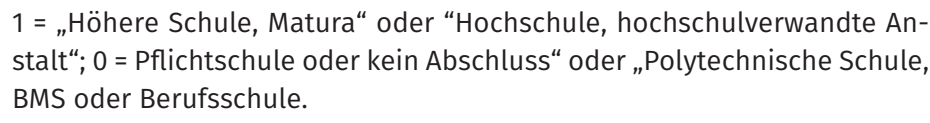 \\
\hline Alter & Vorwahl & Alter in Jahren. \\
\hline Beruf & Vorwahl & 3 Kategorien: „Sonstige“ (Referenz), „Angestellte/r“, „Arbeiter/in“, \\
\hline Erwerbstätigkeit & Vorwahl & $\begin{array}{l}4 \text { Kategorien: „Erwerbstätig“ (Referenz), ,"in Pension“, „Schüler/ } \\
\text { Studierende“, „Sonstige Erwerbstätigkeit“. }\end{array}$ \\
\hline Negative Einkommenssituation & Vorwahl & 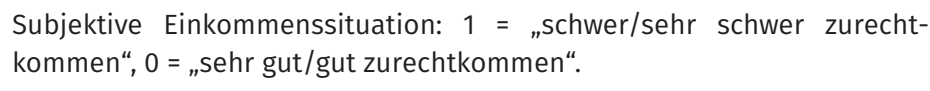 \\
\hline Migrationshintergrund & Vorwahl & $\begin{array}{l}1=\text { beide Elternteile nicht in Österreich geboren, } \\
0=\text { kein Migrationshintergrund. }\end{array}$ \\
\hline Gewerkschaftsmitglied & Vorwahl & $1=\mathrm{ja}, 0=$ nein/sonstige Antworten. \\
\hline Wohngebiet & Vorwahl & $\begin{array}{l}\text { Einschätzung } 3 \text { Kategorien: „Dorf“ (Referenz), „kleine/mittlere Stadt“, } \\
\text { „Großstadt Zentrum/Rand“. }\end{array}$ \\
\hline Subjektive Religiosität & Vorwahl & Dummy (recodiert): 1 = eher/sehr religiös; 0 = überhaupt nicht $/$ nicht sehr. \\
\hline Negative Wirtschaftslage & Vorwahl & $\begin{array}{l}\text { Einschätzung 5-stufig: } 1 \text { = „viel besser geworden“ bis } 5=\text { „viel schlechter } \\
\text { geworden“. }\end{array}$ \\
\hline Demokratiezufriedenheit & $\begin{array}{l}\text { CAPI: Vorwahl/ } \\
\text { CAWI: } \\
\text { Vorwahl/Nach- } \\
\text { wahl }\end{array}$ & $\begin{array}{l}\text { Einschätzung 4-stufig: } 4 \text { = „sehr zufrieden“, } 3 \text { = „eher zufrieden“, } \\
2 \text { = „wenig zufrieden“, } 1 \text { = „gar nicht zufrieden“. }\end{array}$ \\
\hline Politisches Interesse & Vorwahl & $\begin{array}{l}\text { Einschätzung 4-stufig: } 4 \text { = „sehr interessiert“, } 3 \text { = „ziemlich interessiert“, } \\
2 \text { = „wenig interessiert“, } 1 \text { = „gar nicht interessiert“. }\end{array}$ \\
\hline $\begin{array}{l}\text { Ablehnende Einstellung } \\
\text { bzgl. Zuwanderung }\end{array}$ & Vorwahl & $\begin{array}{l}\text { Mittelwert von zwei Items: „Die Österreichische Kultur wird durch Zu- } \\
\text { wanderung bereichert (umgepolt).“ sowie „Die Zuwanderung nach Ös- } \\
\text { terreich soll gestoppt werden." (jeweils 5-Punkt-Skala) ( } \alpha \text { CAPI = .72 bzw. } \\
\alpha C A W I=.77 \text { ) }\end{array}$ \\
\hline $\begin{array}{l}\text { Ablehnende Einstellung } \\
\text { bzgl. Muslimen }\end{array}$ & Vorwahl & $\begin{array}{l}\text { Mittelwert von zwei Items: „Durch die vielen Muslime in Österreich fühle } \\
\text { ich mich manchmal wie ein Fremder im eigenen Land.“ sowie „Der euro- } \\
\text { päische Lebensstil und der von Muslimen sind problemlos vereinbar (um- } \\
\text { gepolt).“ (jeweils 5-Punkt-Skala) ( } \alpha \text { CAPI =.60 bzw. aCAWI =.59) }\end{array}$ \\
\hline Links-Rechts-Skala & Vorwahl & 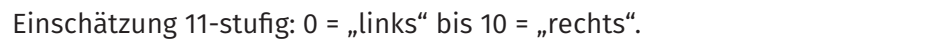 \\
\hline Autoritäre Einstellungen & Vorwahl & $\begin{array}{l}\text { Siehe für Details: Aichholzer/Zeglovits (2015). } \\
\text { Skalenwerte von 1-5. }\end{array}$ \\
\hline
\end{tabular}

Anmerkung: Für Details siehe die Dokumentationen zu Kritzinger et al. 2016a; 2016b 
